\title{
Rare decays at LHCb
}

\author{
Marianna Fontana*† \\ CERN and INFN \\ E-mail: marianna.fontana@cern.ch
}

LHCb is playing a crucial role in the study of rare and forbidden decays of charm hadrons, which might reveal effects beyond the Standard Model. Results on the $D^{0} \rightarrow h^{+} h^{-} \mu^{+} \mu^{-}$and $D^{0} \rightarrow$ $e^{ \pm} \mu^{\mp}$ decays are presented together with some prospects on the LHC future runs.

VIII International Workshop On Charm Physics

5-9 September, 2016

Bologna, Italy

* Speaker.

${ }^{\dagger}$ On behalf of the LHCb collaboration. 


\section{Introduction}

Studies of rare decays of charmed hadrons are a unique tool to probe the Standard Model (SM) couplings for up-type quarks, complementary to similar analyses performed in the beauty and strange sectors. Particularly sensitive to New Physics (NP) contribution are Flavour Changing Neutral Currents (FCNC) and Lepton Number Violating (LNV) decays. In the SM the FCNC processes are suppressed because of the Glashow-Illiopoulus-Maiani (GIM) mechanism, which is particularly effective for $c \rightarrow u$ transitions. On the other hand the LFV decays are completely forbidden in the SM but can be enhanced in some NP scenarios. The branching fraction of $D^{0} \rightarrow$ $h^{+} h^{-} \mu^{+} \mu^{-}$decays is dominated by Long Distance (LD) contribution $\left(\mathscr{O}\left(10^{-6}\right)\right.$ ), when the muons are originating from a resonance, while the Short Distance (SD) contribution are only effective away from the vector meson regions $\left(\mathscr{O}\left(10^{-9}\right)\right)$. In addition to the search studies, multibody rare decays are particularly interesting to study $\mathrm{CP}$ and forward-backward (FB) asymmetries, which could be enhanced by some NP effects up to $\mathrm{O}(1 \%)$ level [1].

The LHCb experiment [2] is the ideal environment to perform these studies, due to the large charm production cross-section $[14,15]$ at the LHC and its unique geometrical acceptance. The detector is a single-arm forward spectrometer with excellent tracking, vertexing and particle identification performance. The trigger system is very flexible and efficient in selecting charmed hadron decays.

\section{Search for the decay $D^{0} \rightarrow \pi^{+} \pi^{-} \mu^{+} \mu^{-}$}

The $D^{0} \rightarrow \pi^{+} \pi^{-} \mu^{+} \mu^{-}$is a FCNC decay, whose branching fraction is dominated by the presence of the $D^{0} \rightarrow \phi\left(\mu^{+} \mu^{-}\right) \pi^{+} \pi^{-}$decay. A first limit on the branching fraction, set by the E791 Collaboration, was $3.0 \times 10^{-5}$ at $90 \%$ CL [16].

The LHCb experiment performed a search [7] using pp collision data sample collected in 2011 and corresponding to an integrated luminosity of $1.0 \mathrm{fb}^{-1}$ at $\sqrt{s}=7 \mathrm{TeV}$. The analysis is performed using the $D^{*+} \rightarrow D^{0} \pi^{+}$decay in order to suppress the combinatorial background. This is further suppressed by means of a multivariate classifier, namely a Boosted Decision Tree (BDT) [3, 4], whose training is performed using as input kinematic and geometric distributions. The main background comes from the misidentification of pions as muons in the decay $D^{0} \rightarrow \pi^{+} \pi^{-} \pi^{+} \pi^{-}$, which can be reduced making stringent requirements on the muon identification. The $\mu^{+} \mu^{-}$invariant mass is divided into four regions to isolate those sensitive to the SD component from those dominated by resonances. The signal regions are defined as $250<m_{\mu \mu}<525 \mathrm{MeV}$ and $m_{\mu \mu}>1100 \mathrm{MeV}$. The $D^{0} \rightarrow \pi^{+} \pi^{-} \phi\left(\mu^{+} \mu^{-}\right)$decay is used as a normalisation channel. Its branching fraction has been derived indirectly from the amplitude analysis of the $D^{0} \rightarrow K^{+} K^{-} \pi^{+} \pi^{-}$decay [8], by taking the fit fraction of the $\pi^{+} \pi^{-} \phi\left(K^{+} K^{-}\right)$and from the $\mathscr{B}\left(\phi \rightarrow \mu^{+} \mu^{-}\right) / \mathscr{B}(\phi \rightarrow$ $\left.K^{+} K^{-}\right)$ratio, which yields to $\mathscr{B}\left(D^{0} \rightarrow \pi^{+} \pi^{-} \phi\left(K^{+} K^{-}\right)\right)=(5.2 \pm 1.1) \cdot 10^{-7}$. This procedure results in a $17 \%$ systematic uncertainty, dominating over the others.

An unbinned two-dimensional maximum likelihood fit is performed to the $m_{\pi \pi \mu \mu}, \Delta m=$ $m_{\pi \pi \mu \mu \pi}-m_{\pi \pi \mu \mu}$ distributions to determine the yields in the ranges $1810-1920 \mathrm{MeV}$ and $140-$ 151.4 MeV as shown in Fig. 1. The yields are compatible with the expected leakage from the resonant into the signal region. A limit on the SD branching fraction, extrapolated to the whole 
di-muon invariant mass spectrum by assuming a phase-space model has been set using the $\mathrm{CL}_{s}$ method (Fig. 2) to:

$$
\mathscr{B}\left(D^{0} \rightarrow \pi^{+} \pi^{-} \mu^{+} \mu^{-}\right)<5.5(6.7) \times 10^{-7} \text { at } 90(95 \%) \mathrm{CL}
$$
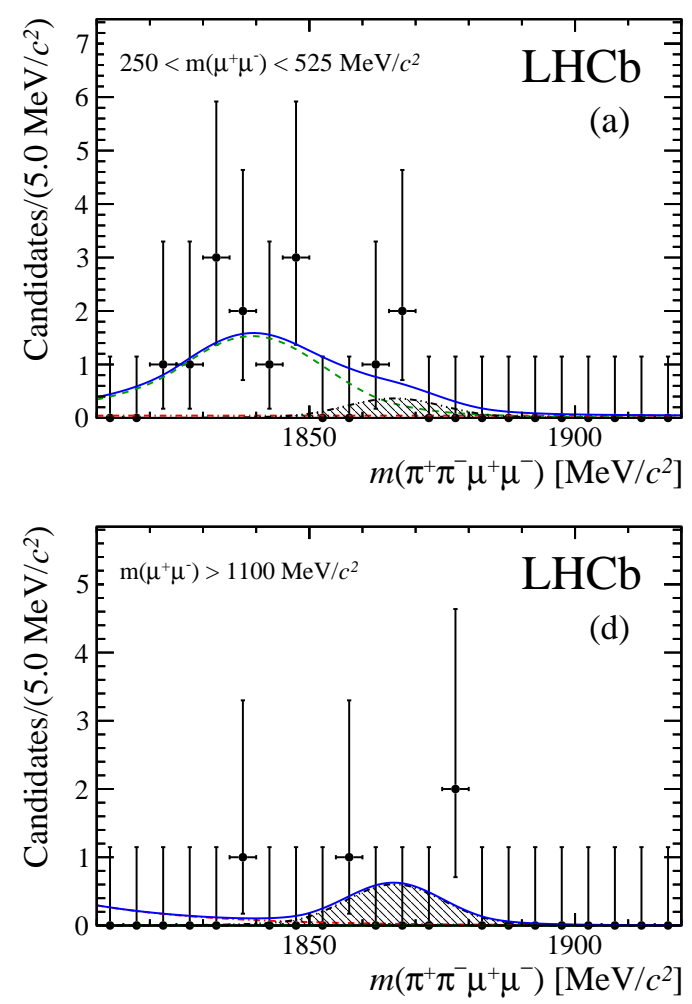
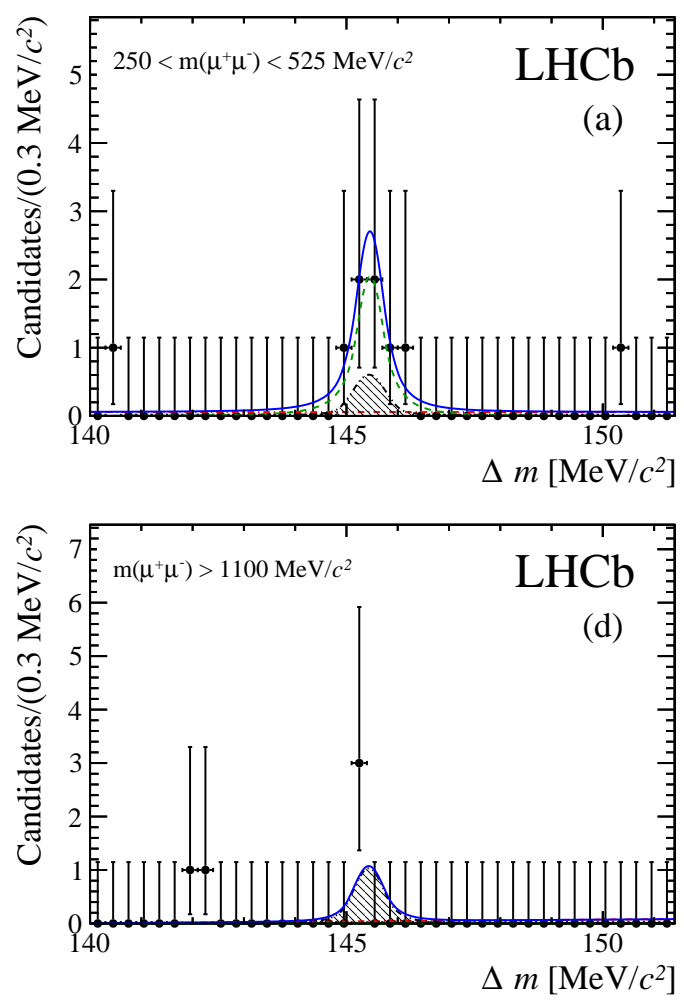

Figure 1: Distribution of $m_{\pi \pi \mu \mu}$ (right) and $\Delta m=m_{\pi \pi \mu \mu \pi}-m_{\pi \pi \mu \mu}$ (left) in the low (top) and high (bottom) $m_{\mu \mu}$ spectra. The data are represented by the points (black) and the fit shape (blue line) is superimposed. The single fit components are the signal (filled area), the misidentified background (dashed green line) and the combinatorial background (dashed-dotted red line).

\section{First observation of the decay $D^{0} \rightarrow K^{-} \pi^{+} \mu^{+} \mu^{-}$in the $\rho^{0} / \omega$ region}

The branching fraction of the $D^{0} \rightarrow K^{-} \pi^{+} \mu^{+} \mu^{-}$decay is dominated by LD contributions, due to presence of intermediate resonance such as the $\rho^{0}$ or $\omega$ mesons. Moreover it is not FCNC and therefore it has limited sensitivity to NP contributions. However these decays can be used to normalise other $c \rightarrow u \mu^{+} \mu^{-}$processes. The LHCb collaboration studied the $D^{0} \rightarrow K^{-} \pi^{+} \mu^{+} \mu^{-}$ decay with the di-moun pair in the $\rho / \omega$ region to provide a suitable normalisation for other 4-body modes such as $D^{0} \rightarrow \pi^{-} \pi^{+} \mu^{+} \mu^{-}$and $D^{0} \rightarrow K^{-} K^{+} \mu^{+} \mu^{-}$. The analysis [10] is based on data collected at a center-of-mass energy of $8 \mathrm{TeV}$, corresponding to an integrated luminosity of $2 \mathrm{fb}^{-1}$. The di-muon invariant mass has been selected in the $\rho^{0}$ and $\omega$ region $\left(675<m_{\mu \mu} \mathrm{MeV}<875\right)$, 


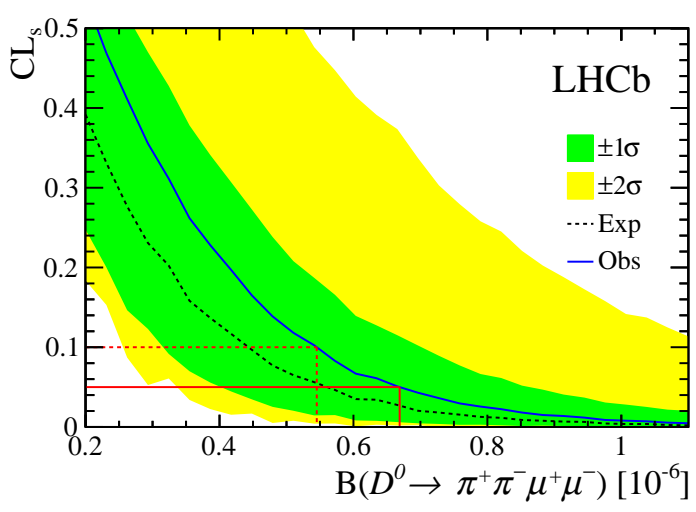

Figure 2: Observed (solid curve) and expected (dashed curve) $\mathrm{CL}_{s}$ values as a function of $\mathscr{B}\left(D^{0} \rightarrow\right.$ $\left.\pi^{+} \pi^{-} \mu^{+} \mu^{-}\right) / \mathscr{B}\left(D^{0} \rightarrow \pi^{+} \pi^{-} \phi\left(\mu^{+} \mu^{-}\right)\right)$. The upper limits at the $90(95) \% \mathrm{CL}$ are indicated by the dashed (solid) line.

which simplify the analysis since no efficiency variation as a function of the di-muon mass is expected.

The main backgrounds components are the misidentified $D^{0} \rightarrow K^{-} \pi^{+} \pi^{+} \pi^{-}$decay, used also as reference channel, and the combinatorial one. The selection on the signal and the normalisation decays makes use of a BDT and differs only for the muon identification requirements. The signal yield is determined with a maximum likelihood fit. The result is shown in Fig. 3, from which the first observation of this decay can be clearly inferred. The corresponding branching fraction is determined using as input also the measurement for the normalisation decay performed by CLEO [9]

$$
\mathscr{B}\left(D^{0} \rightarrow K^{-} \pi^{+} \mu^{+} \mu^{-}\right)=(4.12 \pm 0.12 \pm 0.38) \times 10^{-6},
$$

where the first uncertainty is statistical and the second systematic. This result is in agreement with the theoretical predictions.

The distribution of the $m_{k \pi}$ and $m_{\mu \mu}$ invariant masses are shown in Fig. 4, where the background has been statistically subtracted. The presence of resonant contribution is evident, though a precise understanding of the decay dynamics would require an amplitude analysis.

\section{Search for the decay $D^{0} \rightarrow e^{ \pm} \mu^{\mp}$}

The branching fraction of the LFV decay $D^{0} \rightarrow e^{ \pm} \mu^{\mp}$ is predicted to be non-zero in some beyond the SM theories [11] and its observation with current sensitivities would be a clear sign of NP. The most stringent limit was set by Belle [12] at

$$
\mathscr{B}\left(D^{0} \rightarrow e^{ \pm} \mu^{\mp}\right)<2.6 \times 10^{-7} \text { at } 90 \% \mathrm{CL} .
$$

The LHCb experiment performed a measurement using the data of the entire sample of LHC Run 1 corresponding to an integrated luminosity of $3 \mathrm{fb}^{-1}$. The analysis is based on the $D^{*+} \rightarrow$ $D^{0} \pi^{+}$decay chain and the $D^{0} \rightarrow K^{-} \pi^{+}$channel is used as normalisation. The most important peaking background is the $D^{0} \rightarrow \pi^{+} \pi^{-}$decay, that can be misidentified as $D^{0} \rightarrow e^{ \pm} \mu^{\mp}$ with a probability of about $1.4 \times 10^{-8}$. The peak of this background is just $15 \mathrm{MeV}$ below the signal, as shown 

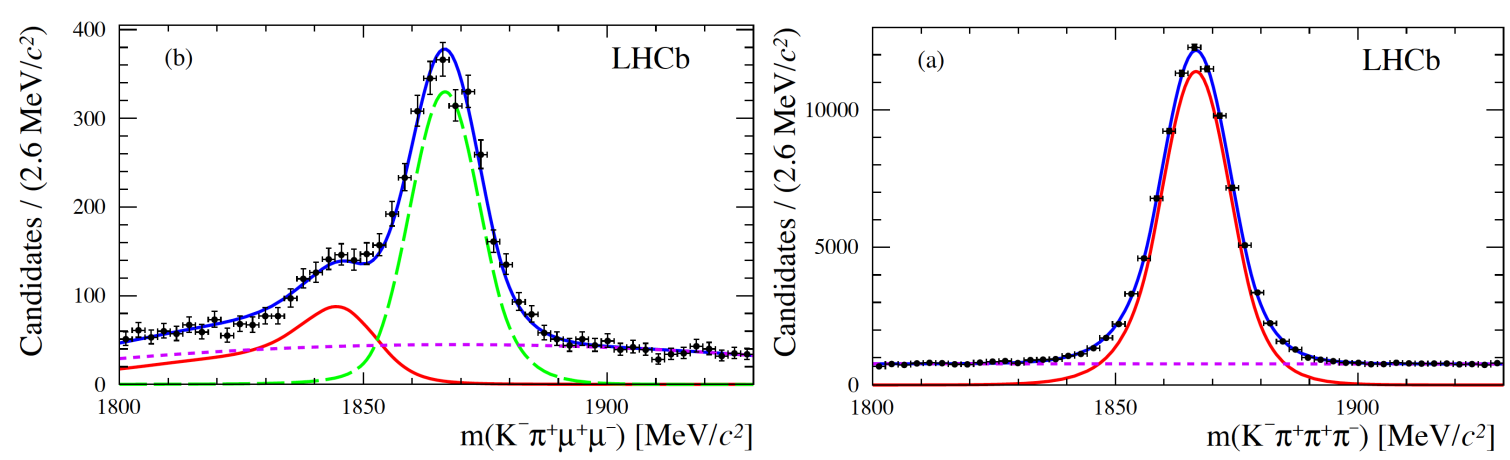

Figure 3: Distribution of $m_{K \pi \mu \mu}$ (Left) and $m_{K \pi \pi \pi}$ (Right). The data are represented with the points (black) and the fit function (blue) is overlaid. The $D^{0} \rightarrow K^{-} \pi^{+} \mu^{+} \mu^{-}$(green dashed line), the $D^{0} \rightarrow K^{-} \pi^{+} \pi^{+} \pi^{-}$ (red solid line) and the combinatorial background (magenta dotted line) are also shown.
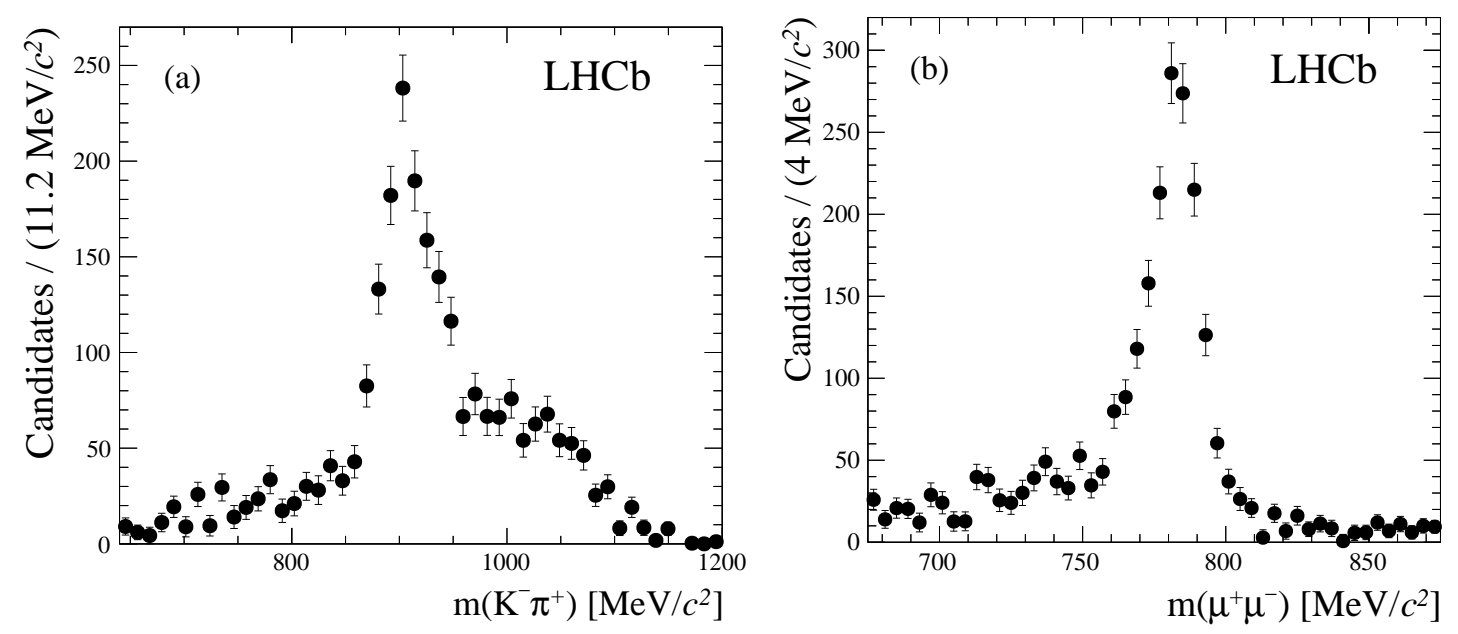

Figure 4: Background subtracted distribution of the $m_{p i K}$ (Left) and $m_{\mu \mu}$ (Right) for selected $D^{0} \rightarrow$ $K^{-} \pi^{+} \mu^{+} \mu^{-}$candidates.

in Fig. 5. Moreover the parametrisation of the signal is particularly difficult, due to bremsstrahlung effects, which have to be taken into account in the correction of the electron momentum. As usual the combinatorial background is suppressed using a BDT.

In order to enhance the sensitivity to the signal the sample has is divided into BDT bins, in which the number of signal events is extracted simultaneously using an unbinned maximum likelihood fit. The invariant mass distribution for the most sensitive BDT bin is shown in Fig. 6 . The yield is compatible with zero and the upper limit has been set with the $\mathrm{CL}_{s}$ method (Fig. 4)

$$
\mathscr{B}\left(D^{0} \rightarrow e^{ \pm} \mu^{\mp}\right)<1.3(1.6) \times 10^{-8} \text { at } 90(95 \%) \mathrm{CL} .
$$

\section{Future prospects}

The measurements presented in the previous sections have been performed with the data collected during the Run 1 of LHC. An important measurement that is currently in progress with the 


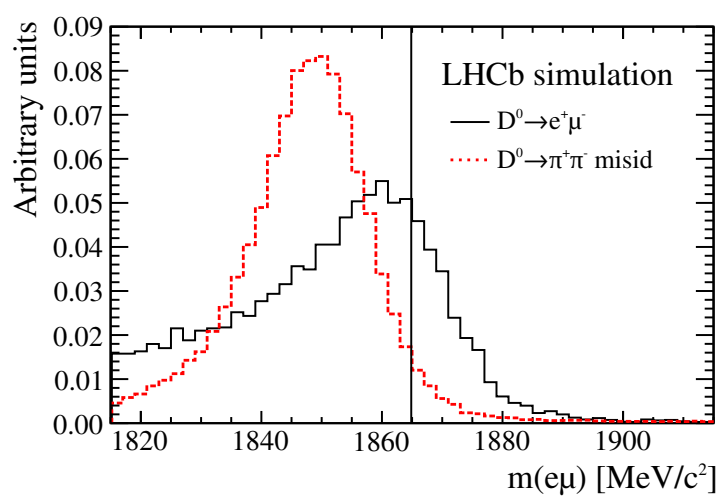

Figure 5: Invariant mass from simulation for $D^{0} \rightarrow e^{ \pm} \mu^{\mp}$ (black solid line) and $D^{0} \rightarrow \pi^{ \pm} \pi^{\mp}$ decays reconstructed as $D^{0} \rightarrow e^{ \pm} \mu^{\mp}$ (red dashed line).
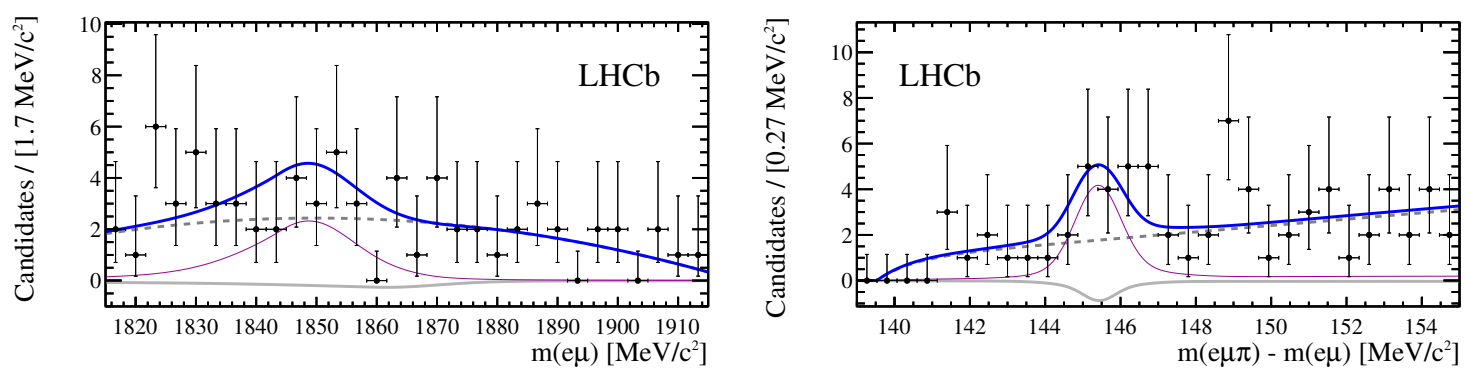

Figure 6: Distribution of $m_{e \mu}$ (left) and $m_{e \mu \pi}-m_{e \mu}$ for $D^{0} \rightarrow e \mu$ candidates with fit functions overlaid, for the most signal-like BDT bin. The total fit results (blue solid line), the $D^{0} \rightarrow e \mu$ (grey line), the misidentified $D^{0} \rightarrow \pi \pi$ (grey dashed line) and the combinatorial background (purple line) components are also shown.

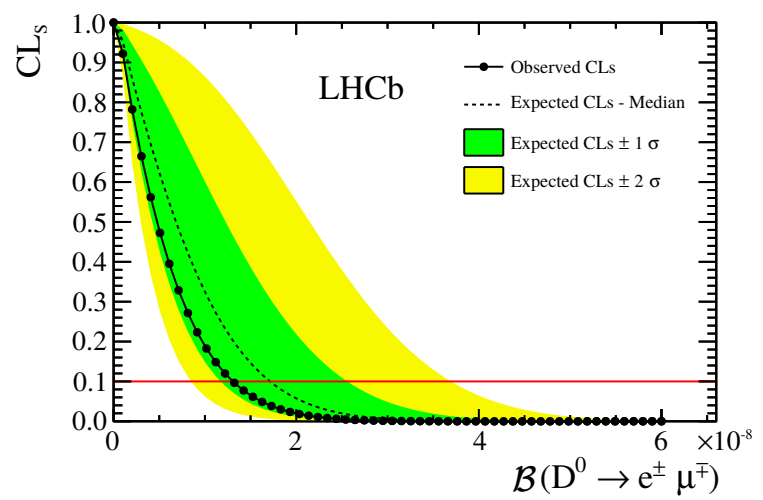

Figure 7: Distribution of $\mathrm{CL}_{\mathrm{s}}$ as a function of $\mathscr{B}\left(D^{0} \rightarrow e^{ \pm} \mu^{\mp}\right)$. The expected distribution is shown by the dashed line. The observed distribution is shown by the solid line connecting the data points. The horizontal line indicates the $90 \% \mathrm{CL}$. 
same sample is the update on the search for the FCNC decay $D^{0} \rightarrow \mu^{+} \mu^{-}$.

At present LHC started a second phase of data taking, at the end of which the LHCb detector will have collected about $8 \mathrm{fb}^{-1}$ of integrated luminosity. Moreover for the long-term future $\mathrm{LHCb}$ is planning an upgrade of the detector, and it is expected to collect an additional $50 \mathrm{fb}^{-1}$. Assuming the same efficiencies and signal to background ratios as in Run 1 it is possible to give some estimate on the expected branching fractions for some decays. The values, reported in Tab. 5, show that after the Upgrade it will be possible, for some modes, to reach the sensitivities at the level of NP predictions.

\begin{tabular}{lcc}
\hline Mode & Run 2 & Upgrade 1 \\
\hline$D^{0} \rightarrow \mu^{+} \mu^{-}$ & $\sim 10^{-9}$ & $\sim 10^{-10}$ \\
$D^{+} \rightarrow \pi^{+} \mu^{+} \mu^{-}$ & $\sim 10^{-8}$ & $\sim 10^{-9}$ \\
$D_{s}^{+} \rightarrow K^{+} \mu^{+} \mu^{-}$ & $\sim 10^{-7}$ & $\sim 10^{-8}$ \\
$D^{0} \rightarrow h^{+} h^{-} \mu^{+} \mu^{-}$ & $\sim 10^{-7}$ & $\sim 10^{-8}$ \\
\hline
\end{tabular}

Table 1: Extrapolation to Run 2 and Upgrade 1 luminosity on the branching fraction of some FCNC charm decays.

Moreover, $\mathrm{CP}$ and FB asymmetries can be also investigated at the percent level as reported in Tab. 5. The predictions could be further improved thanks to better selections and a novel trigger strategy entirely software based.

\begin{tabular}{lll}
\hline Mode & Run 2 & Upgrade 1 \\
\hline$D^{+} \rightarrow \pi^{+} \mu^{+} \mu^{-}$ & $0.6 \%$ & $0.2 \%$ \\
$D^{0} \rightarrow \pi^{+} \pi^{-} \mu^{+} \mu^{-}$ & $3 \%$ & $1 \%$ \\
$D^{0} \rightarrow K^{-} \pi^{+} \mu^{+} \mu^{-}$ & $1 \%$ & $0.3 \%$ \\
$D^{0} \rightarrow K^{+} \pi^{-} \mu^{+} \mu^{-}$ & $40 \%$ & $12 \%$ \\
$D^{0} \rightarrow K^{-} K^{+} \mu^{+} \mu^{-}$ & $11 \%$ & $4 \%$ \\
\hline
\end{tabular}

Table 2: Extrapolation to Run 2 and Upgrade 1 luminosity on asymmetries of some FCNC charm decays.

\section{Conclusion}

The results presented show excellent prospects for rare charm decays at LHCb. The Run 1 measurements improved with respect to the previous ones in some cases by several orders of magnitude. The LHCb collaboration is putting in place an upgrade of the detector, that will allow to collect a sample two orders of magnitude bigger. The collected statistics would be enough in various cases to reach the level of NP theoretical predictions. Moreover measurement on CP and FB asymmetries are expected to reach the percent level.

\section{References}

[1] L. Cappiello, O. Cata and G. D’ Ambrosio, JHEP 1304 (2013) 135 doi:10.1007/JHEP04(2013)135 [arXiv:1209.4235 [hep-ph]]. 
[2] R. Aaij et al. [LHCb Collaboration], JINST 3 (2008) S080005.

[3] L. Breiman, J. H. Friedman, R. A. Olshen, and C. J. Stone, Wadsworth international group, Belmont, California, USA, 1984.

[4] R. E. Schapire and Y. Freund, J. Comput. Syst. Sci. 55 (1997) 119.

[5] R. Aaij et al. [LHCb Collaboration], Nucl. Phys. B 871(2013) 1-20 doi:10.1016/j.nuclphysb.2013.02.010 [arXiv:1302.2864 [hep-ph]]

[6] R. Aaij et al. [LHCb Collaboration], JHEP 03 (2016) 159, doi:10.1007/JHEP03(2016)159 [arXiv:1510.01707 [hep-ph]]

[7] R. Aaij et al. [LHCb Collaboration], Phys. Lett. B 728 (2014) 234 doi:10.1016/j.physletb.2013.11.053 [arXiv:1310.2535 [hep-ex]].

[8] M. Artuso et al. [CLEO Collaboration], Phys. Rev. D 85 (2012) 122002 doi:10.1103/PhysRevD.85.122002 [arXiv:1201.5716 [hep-ex]].

[9] G. Bonvicini et al. [CLEO Collaboration], Phys. Rev. D 89 (2014) 072002 doi:10.1103/PhysRevD.89.072002 [arXiv:1312.6775 [hep-ex]].

[10] R. Aaij et al. [LHCb Collaboration], Phys. Lett. B 757 (2016) 558 doi:10.1016/j.physletb.2016.04.029 [arXiv:1510.08367 [hep-ex]].

[11] G. Burdman, E. Golowich, J. Hewett, and S. Pakvasa, Phys. Rev. D 66, 014009 doi:10.1103/PhysRevD.66.014009 [arXiv:0112235 [hep-ph]].

[12] M. Petric et al. [Belle Collaboration], Phys. Rev. D 81 (2010) 091102 doi:10.1103/PhysRevD.81.091102 [arXiv:1003.2345 [hep-ex]].

[13] R. Aaij et al. [LHCb Collaboration], Phys. Lett. B 754 (2016) 167 doi:10.1016/j.physletb.2016.01.029 [arXiv:1512.00322 [hep-ex]].

[14] R. Aaij et al. [LHCb Collaboration], Nucl. Phys. B 871 (2013) 1 doi:10.1016/j.nuclphysb.2013.02.010 [arXiv:1302.2864 [hep-ex]].

[15] R. Aaij et al. [LHCb Collaboration], JHEP 1603 (2016) 159 Erratum: [JHEP 1609 (2016) 013] doi:10.1007/JHEP03(2016)159, 10.1007/JHEP09(2016)013, 10.1007/JHEP03(2016)159 10.1007/JHEP09(2016)013 [arXiv:1510.01707 [hep-ex]].

[16] E. M. Aitala et al. [Fermilab E791 Collaboration] Phys. Rev. Lett. 86, 3969 